\title{
Near-field enhancement and imaging in double cylindrical polariton-resonant structures: Enlarging perfect lens
}

\author{
Pekka Alitalo, Stanislav Maslovski, and Sergei Tretyakov \\ Radio Laboratory / SMARAD, Helsinki University of Technology \\ P.O. Box 3000, FI-02015 TKK, Finland \\ E-mails: pekka.alitalo@tkk.fi,stanislav.maslovski@tkk.fi,sergei.tretyakov@tkk.fi
}

(Dated: November 12, 2018)

\begin{abstract}
We experimentally demonstrate a prototype of a cylindrical enlarging lens capable of enhancing and restoring evanescent fields. The enabling phenomenon is the resonant excitation of coupled surface modes in a system of two cylindrical arrays of small resonant particles. As was shown in [J. Appl. Phys. 96, 1293 (2004)], this phenomenon in planar arrays can be used in electromagnetic near-field imaging. Here, we use a similar structure in a cylindrically symmetric configuration, which gives us a possibility to obtain an enlarged near-field image.
\end{abstract}




\section{INTRODUCTION}

Since J. Pendry found that a slab filled by a material with negative parameters $\varepsilon$ and $\mu$ theoretically operates as a perfect lens [1], enhancing evanescent components, many researchers tried to realize such a device experimentally. The main difficulty in the design is the problem of losses in the artificial material filling the lens. Recently, we proposed an alternative approach to enhancement and imaging of evanescent fields, which is based on two parallel planar lattices of small resonant particles [2]. Since no volumetric artificial media are used in this device, the issue of losses can be mitigated. Experiments in the microwave region have demonstrated imaging of near fields in these new structures [2]. Evanescent fields are enhanced on lattices of small resonant particles, because these planar structures support propagation of slow electromagnetic waves, which is also the key property of interfaces between vacuum and materials with negative parameters. Such waves are known also as surface or Zenneck waves in radio science, or as surface plasmons or polaritons in optics. These waves are called slow because their phase velocity (along the surface) is less than the speed of light. Respectively, the fields of such waves decay exponentially from the surface.

The ideal planar perfect lens restores the field distribution of the source (both amplitude and phase) in the image plane. A natural extension would be the use of more complex lens shapes to enable also enlargement of the image. Using geometrical optics, it has been theoretically shown that image enlargement is indeed possible in a perfect lens of a circular cylindrical shape [3]. However, no attempts to realize this effect using volumetric metamaterials experimentally have been made.

Our purpose here is to experimentally show enhancement of evanescent fields and enlarged images of source field distribution using arrays of resonant particles. For this purpose we construct two concentric lattices of particles, creating a cylindrically symmetrical system. We restrict ourselves by the case when the surface modes propagate around the cylinders, i.e. we consider only the angular spatial spectrum. Contrary to the infinite planar geometries this spectrum is discrete.

To excite slow waves, either a non-uniformity in the surface is needed, or the incident field must be in phase synchronism with these waves. The latter means that the exciting field must be an evanescent plane wave. When the propagation factor (along the surface) of the incident wave coincides with the propagation factor of a surface wave, a strong resonance 
occurs. In this case the surface wave is most effectively excited.

The source of evanescent field and the cylindrical arrays are placed in a model cell de-

scribed in [2]. Essentially, this cell is a planar waveguide operating below cut-off. Because of the symmetry of the waveguide modes, a circular ring of particles placed inside the waveguide behaves as an infinitely long cylinder under incidence of an evanescent wave. We will show that the surface modes excited on the arrays can be used to amplify near fields of a source and, respectively, to obtain an image mimicking the distribution of the source field on a larger scale.

\section{EXPERIMENT}

The experimental setup was similar to that used in [2]. The setup consisted of a two-plate waveguide, in which the distance between the metal plates $(h)$ was smaller than $\lambda / 2(\lambda$ is the wavelength in free space). The setup is shown in Fig. 1]. The evanescent source fields were excited by two horizontal $\lambda / 4$-dipoles placed inside the waveguide. The operating frequency of the setup was close to $5 \mathrm{GHz}$ and therefore $h$ was chosen to be $2.5 \mathrm{~cm}(\lambda$ at $5 \mathrm{GHz}$ is 6 $\mathrm{cm})$.

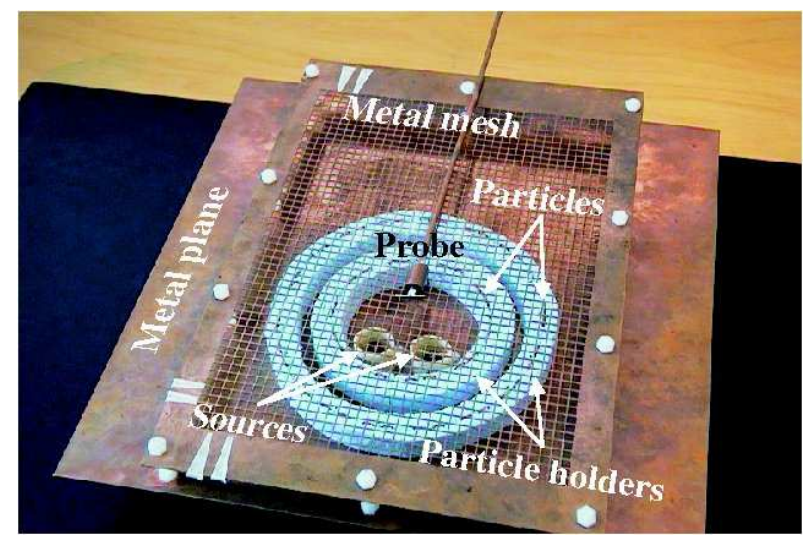

FIG. 1: (Color online). Experimental setup used to measure the distribution of the evanescent fields inside the waveguide.

In order to measure the fields inside the waveguide, the upper plate of the waveguide was made of a dense metal wire mesh, which was weakly penetrable to the fields inside the waveguide (the transmission coefficient of the mesh was measured to be about $-20 \mathrm{~dB}$ for normal plane-wave incidence at $5 \mathrm{GHz}$ ). The distribution of electric field inside the 
waveguide could then be measured using a probe, which was a horizontal $\lambda / 4$-dipole placed approximately $3 \mathrm{~mm}$ above the metal mesh and controlled by a robot connected to a PC.

The source dipoles were connected to port 1 of a vector network analyzer (VNA) using a power divider and the probe was connected to port 2 of the VNA. By scanning the surface of the metal mesh with the probe and at the same time measuring $S_{21}$ with the VNA, it was possible to measure the relative field distribution (in the horizontal plane) inside the waveguide.

The cylindrical arrays consisted of small particles. These particles, in which the surface resonance occurs, were made of thin copper wires that were meandered to make the overall particle size considerably smaller than the resonant wavelength, see Fig. 2, First a large amount of these resonant particles was manufactured and each was tested to have approximately the same resonant frequency $\left(f_{r} \approx 5.464 \mathrm{GHz}\right)$. The testing was done by placing the particle under test inside the waveguide and close to a source dipole (distance between the particle and the source dipole was approximately $2 \mathrm{~cm}$ ). When the field magnitude was measured as described before, the field on top of the resonant particle had a sharp peak (much stronger than the field on top of the source dipole) at a certain frequency. This frequency was then determined to be approximately the same for all the resonant particles used.

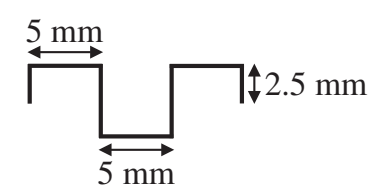

FIG. 2: A resonant particle with $f_{r} \approx 5.464 \mathrm{GHz}$. Diameter of the copper wire is $0.8 \mathrm{~mm}$.

First an array consisting of ten resonant particles and a radius of $r=40 \mathrm{~mm}$ was constructed. The array was placed inside the waveguide around two source dipoles (the dipoles were approximately in the center between the two plates of the waveguide) with the other end of the array about $15 \mathrm{~mm}$ from the dipoles. The resonant particles were placed in a dielectric foam holder $\left(\varepsilon_{r} \approx 1\right)$, so that the particles were approximately in the center between the waveguide's two plates.

When the fields were measured, two strong field maxima occurring at the measuring frequency of $f \approx 5.28 \mathrm{GHz}$ were observed on top of the array, see Fig. 3. The fields in Fig. 3] were measured with the probe dipole oriented along the $x$-axis. By measuring the same 
situation with the probe oriented along the $y$-axis, it was seen that the field distribution was approximately the same as shown in Fig. 3 (the two maxima were at the same places and no other maxima were seen). As can be seen from Fig. 3. the array was strongly excited near the two sources. Also another resonant frequency was found ( $f \approx 5.37 \mathrm{GHz})$ at which also the other side of the array was excited (symmetrically with that shown in Fig. 3, i.e. four maxima altogether).

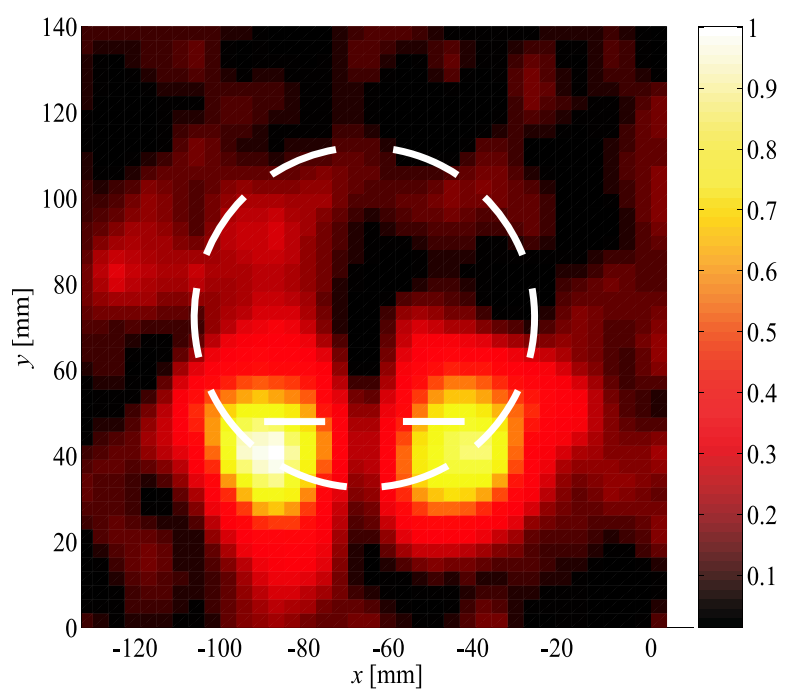

FIG. 3: (Color online). Distribution of electric field amplitude measured on top of the waveguide $(f=5.28 \mathrm{GHz})$. The probe dipole is oriented along the $x$-axis. Two evanescent sources are placed inside a cylindrical array made of resonant particles. The places of the sources and the array are shown with white color.

Next, another, larger array consisting of 18 resonant particles arranged at a circle with the radius $r=70 \mathrm{~mm}$ was manufactured. The measurements showed that this array had many resonant frequencies, with one of them most pronounced. By measuring the field distribution as before (with the other side of the array close to the two sources, as in Fig. 33), it was noticed that there existed a resonance near 5.3 GHz. By manually shifting the positions of the particles, the resonant frequency of the array could be tuned to coincide with the resonant frequency of the smaller array. The array was tuned to resonate at $f=5.28 \mathrm{GHz}$, corresponding to the resonant frequency at which the fields in Fig. 3 were measured.

The particles of the larger array were excited only near the two sources when measured at this frequency. Again two maxima were seen on top of the array. Next, both arrays were 
placed around the sources in a coaxial manner, see Fig. 4. The geometry of this configuration is the same as for a volumetric perfect lens theoretically studied in [3]. From the field plot it is seen that the smaller array was weakly excited and the larger array had four strong field maxima (images of the source field), as shown in Fig. 4. Similar results were obtained with planar arrays in 2], where the resonant array situated closer to the source was not excited at all, but the resonant array situated further had a very strong maximum.

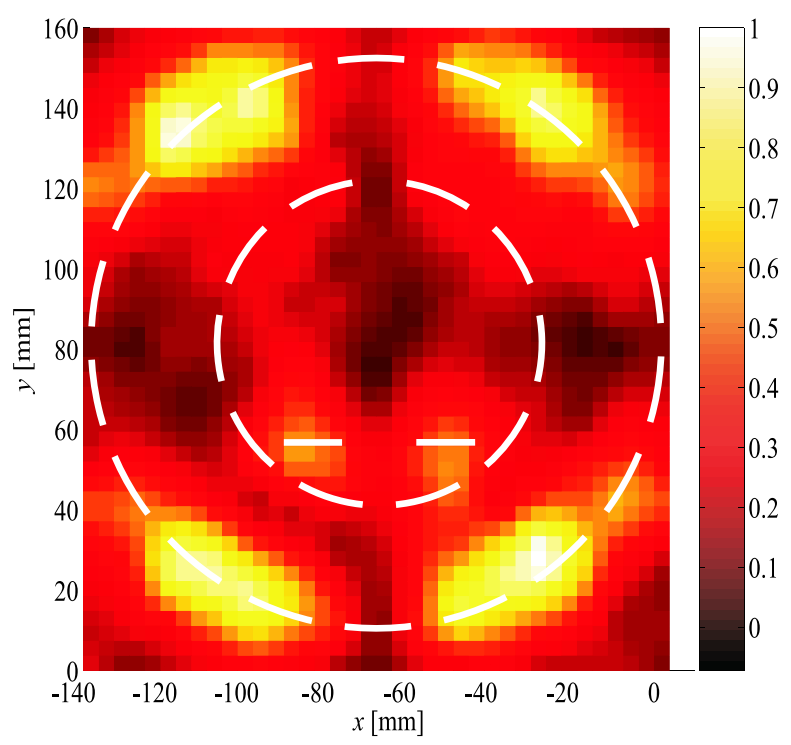

FIG. 4: (Color online). Distribution of electric field amplitude measured on top of the waveguide $(f=5.28 \mathrm{GHz})$. The probe dipole is oriented along the $x$-axis. Two evanescent sources are placed inside two coaxial cylindrical arrays made of resonant particles. The places of the sources and the arrays are shown with white color.

The maxima of the field at the image side appear to follow the same angular distribution as that of the source field. The two images on the other side of the larger array can be considered as "parasitic" images. The fields in Fig. 4 were measured with the probe dipole oriented along the $x$-axis. By measuring the same situation with the probe oriented along the $y$-axis, it was seen that the field distribution was approximately the same as shown in Fig. 4 (the four maxima were at the same places and no other maxima were seen).

The larger array was also tuned to match the other resonant frequency of the smaller array (i.e. $f=5.37 \mathrm{GHz}$ ). Using that frequency, the two "parasitic" images seen in Fig. 4 did not appear. 


\section{CONCLUSION}

We have experimentally demonstrated a prototype of an enlarging perfect lens, using amplification of evanescent fields on two cylindrical arrays of small particles. The arrays supported surface waves at certain frequencies. By tuning the arrays to resonate at the same frequency, the evanescent source fields inside the two arrays were amplified and reached the maximum values on the outer array. According to the measured field distributions, the angular distribution of the source field located inside the two coaxial arrays is properly restored on the outer array. The angular resolution of the image is mainly determined by the period of the arrays. Resonant meandered pieces of copper wires were successfully used in this microwave concept demonstration. For higher-frequency realizations other small resonating particles may be used, such as plasmonic nano-spheres for potential optical applications.

[1] J.B. Pendry, Phys. Rev. Lett. 85, 3966 (2000).

[2] S.I. Maslovski, S.A. Tretyakov, and P. Alitalo, J. Appl. Phys. 96, 1293 (2004).

[3] J.B. Pendry, Optics Express 11, 755 (2003). 